\title{
High capacity and access rate, data storage using laser communications
}

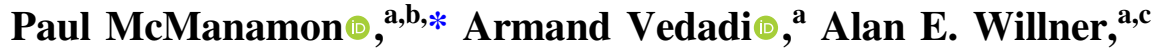 \\ Dipayan Choudhary, ${ }^{a}$ Nick Montifiore, ${ }^{a}$ and Ohad Harlev ${ }^{\text {a }}{ }^{a}$ \\ ${ }^{a}$ Lyteloop, Great Neck, New York, United States \\ ${ }^{b}$ Exciting Technology LLC, Dayton, Ohio, United States \\ ${ }^{c}$ University of Southern California, Los Angeles, California, United States
}

\begin{abstract}
The amount of data in the world is expanding exponentially. About $90 \%$ of the world's data has been created in the last two years, and almost 5\% percent of the world's power is used for data storage and computing. Lyteloop has an approach to data storage that uses optical communications to store data in motion between objects. Since the speed of light is fast, one needs very high data rates and very long paths to store large amounts of data. Data storage of petabytes, and even exabytes, is reasonable with this approach, in a format more compact than traditional data storage methods. LyteLoop has demonstrated an operating system using fiber, and currently has a system operating using free space. The fiber system stores $>1$ Gbyte, using $2000 \mathrm{~km}$ of fiber, and allows us to develop data center-like writing and retrieval experiences. Multiple patents have been filed with some granted. One of the patented concepts is called angle multiplexing, a method of dramatically increasing path length over a free-space path among multiple optical apertures. This has been demonstrated in free space using two different approaches. LyteLoop can store data in fiber, and in free space, either in a vacuum or in space. (C) The Authors. Published by SPIE under a Creative Commons Attribution 4.0 Unported License. Distribution or reproduction of this work in whole or in part requires full attribution of the original publication, including its DOI. [DOI: 10.1117/1.OE.60.1.015105]
\end{abstract}

Keywords: hyper data center; big data; data center; data in motion.

Paper 20200946 received Aug. 8, 2020; accepted for publication Dec. 23, 2020; published online Jan. 11, 2021.

\section{Introduction}

This paper explores the concept of storing large amounts of data in motion during the introduction. ${ }^{1,2}$ Then discusses enabling technologies in Sec. 2, experiments to date in Sec. 3, and conclusions in Sec. 4.

The dawn of the big data $e^{1,2}$ and the boom in analytics-driven industries with machine learning and artificial intelligence pose new challenges in the field of data storage, ${ }^{3-5}$ including physical infrastructure, base and operating costs, and cyber security. ${ }^{6-8}$ Nearly $90 \%$ of the world's data today has been created in the past two years, highlighting the exponential trajectory of data storage requirements. LyteLoop LLC has introduced a revolutionary approach to store large amounts of data in motion, recirculating an optical beam in a loop between satellites, in optical fiber, in a small cell, or in a large vacuum cavity called a tube. ${ }^{9,10}$ The Lyteloop concept creates an optical loop, where data are continuously recirculated and accessed on demand. The speed of light is fast, $c=3 \times 10^{8} \mathrm{~m} / \mathrm{s}$ in vacuum, so storing large quantities of data requires very high data rates and very long path lengths. Specifically, the total capacity is a product of the data rate (in bits per second) and the time $t=$ path length $/ c$, it takes light to complete a single roundtrip (in seconds):

$$
\text { Capacity }=\frac{\text { data rate } \times \text { path length }}{\mathrm{c}},
$$

where the speed of light, $c$, is a function of the refractive index, $n$ (unitless) of the medium. Equation (1) shows capacity is a function of both path length and data rate.

*Address all correspondence to Paul McManamon, paul@excitingtechnology.com 
To have a very high data rate, first consider wavelength multiplexing. Optical $C$ band is the most common lasercom band. This is from 1530 to $1565 \mathrm{~nm} .{ }^{3}$ This covers $3.75 \mathrm{THz}$ of bandwidth. Incorporating the $O, E, S, C, L$, and $S$ bands covers from 1260 to $1675 \mathrm{~nm}$, or $59 \mathrm{THz}$ of bandwidth. The full width of transmission for most fiber would be $\sim 975$ to $1675 \mathrm{~nm}$ or $129 \mathrm{THz}$, but in free space in a vacuum, with no attenuation, 300 to $1700 \mathrm{~nm}$, or $824 \mathrm{THz}$, is possible. Dividing these large bandwidths into $50 \mathrm{GHz}$ regions, provides $75,1180,2572$, and 16,471 channels, respectively, assuming single side-band operation, which will closely pack the lasercom frequency lines. One of the complexity issues is these large numbers. Imagine having to make 16,471 laser lines, separated by $50 \mathrm{GHz}$ each. Individual laser lines can be used or a comb of lines, but there is a need to make a large number of laser lines. Data rates in a single channel can be limited by modulator bandwidths, i.e., the speed at which data is encoded, and the number of gray scales, or bits per symbol period, used [i.e., quadrature phase shift keying (QPSK) versus 16 quadrature amplitude modulation (QAM) versus $64 \mathrm{QAM}$ ]. Increasing the number of bits per symbol, or gray scales comes at a price in power. The signal-to-noise ratio (SNR) required is proportional as given in Eq. (2).

$$
\mathrm{SNR} \propto 2^{C}+1
$$

where $C$ is the number of bits used to define gray levels. Table 1 indicates the relationship between power and \# of bits controlling gray levels.

The required power is almost doubled each time one more bit of controlling gray levels is added. Many commercial lasercom devices provide higher bandwidth by adding gray levels. More bits using gray levels require more power at an exponential relationship. Certainly, doubling 1 to 2 is not as costly in power as doubling 1000 to 2000. It is possible to store $10 \times$ the amount of data in exchange for an increase of $1000 \times$ in required power.

Second, polarization multiplexing doubles the number of channels using two orthogonal states.

The third dimension considered in this work is spatial multiplexing. It is possible to incorporate orbital angular momentum (OAM) or Hermite-Gaussian (HG) beams ${ }^{11,12}$ These are circular or rectangular orthogonal modes, respectively. Any orthogonal basis set will work. OAM beams form doughnut intensity profiles, where the diameter of the circular profile increases with higher OAM order. Additionally, at higher order, these modes become increasingly more difficult to differentiate, and more importantly, diverge faster with propagation distance. The fullwidth-half-maximum (FWHM) beam divergence, $\vartheta$, based on the diffraction limit from a single aperture emitting a Gaussian beam, depends on wavelength $\lambda$ and aperture diameter D:

Table 1 Power ratio vs \# of bits controlling gray levels.

\begin{tabular}{lc}
\hline \hline \multicolumn{2}{c}{ Power required versus gray level } \\
\hline$\#$ of bits controlling gray levels & Ratio of power increase \\
\hline 1 & 1 \\
2 & 3 \\
3 & 7 \\
4 & 15 \\
5 & 31 \\
6 & 63 \\
7 & 127 \\
8 & 255 \\
9 & 511 \\
10 & 1023 \\
\hline \hline
\end{tabular}




$$
\vartheta \approx \frac{\lambda}{D}
$$

The beam waist for OAM modes is given as

$$
w(z)=w_{0}(z) * \sqrt{2 m+|L|+1}
$$

where $L$ is the OAM mode. If we ignore $m$, which has to do with modes going out from the center of the beam, the beam width at any given range will be increased by the square root of the OAM mode \#. We can therefore modify Eq. (4) to be: ${ }^{4}$

$$
\vartheta \approx \frac{\lambda \sqrt{|L|}}{D} .
$$

While spatial multiplexing allows increased channel counts, various practical concerns arise as a result of the non-Gaussian spatial intensity distribution. Gaussian intensity profiles simply require detecting the on-axis field, where most of the intensity is located. The intensity of OAM modes is higher away from the optical axis with increased mode order. This is shown in Fig. 1, in which modes are shown approximately to scale. It is noteworthy that even the first-order OAM modes have zero intensity on-axis, and by the tenth order, nearly all the intensity lies at the edge of the spatial beam. Thus, decoding information carried by OAM modes requires the detection of the full spatial distribution of the beam, which entails the use of significantly larger optical apertures.

Shorter wavelengths have lower beam divergence according to Eq. (5), allowing the use of more OAM modes for a given aperture size. Because of the increased beam divergence with high OAM modes in free space, we will use more OAM modes at shorter wavelengths. Shorter wavelengths not only have a higher density of frequency for a certain wavelength range, but the beam divergence is lower, so more OAM modes can be used for a given aperture size, as shown in Eq. (5). We postulate we can use up to 49 OAM modes for spatial multiplexing or 24 OAM modes on either side of a central mode. The maximum number of OAM modes will depend on being able to differentiate between closely spaced modes. Figure 2 shows an example of how the number of OAM modes might vary by wavelength for a system covering 266 to $1700 \mathrm{~nm}$, with an artificial maximum number of modes set at 49 .

For the fiber case, we can use multiple fiber cores as well as spatial multiplexing. Fibers with multiple cores are becoming available. ${ }^{5}$ Multimode fibers have been available for a long time, and are often easier to make than single-mode fibers. The issue with fibers is controlling the modes in some orthogonal basis set.

The next aspect to maximizing capacity in Eq. (1) is to increase the total roundtrip time or path length of the loop. The simplest form of a loop consists of reflecting a beam back and forth

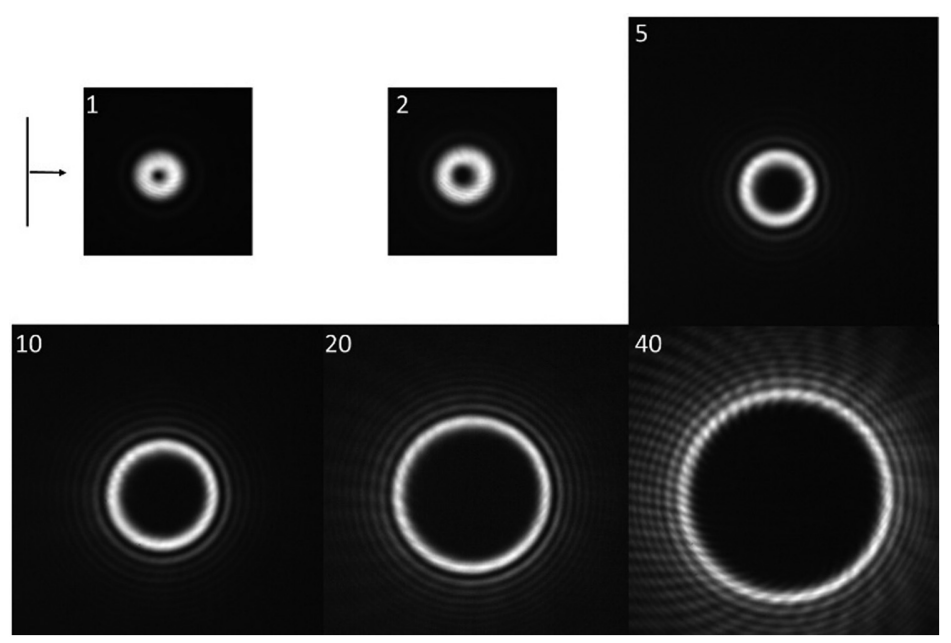

Fig. 1 Intensity distribution of OAM modes. 


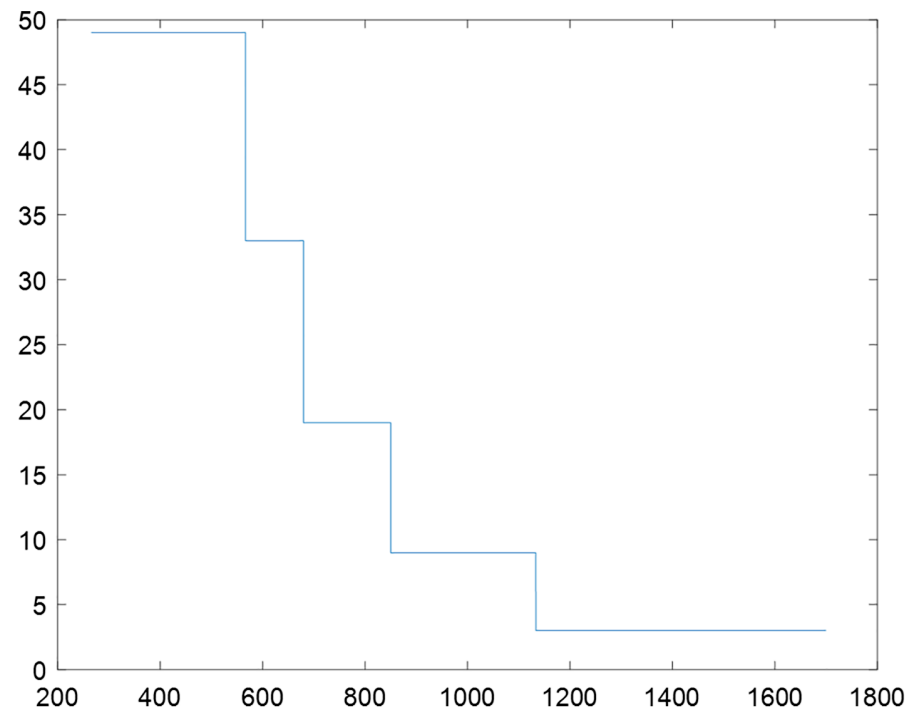

Fig. 2 Number of OAM modes versus wavelength for a certain aperture diameter.

between two apertures (e.g., mirrors). Astronomical distances would be required to sustain high data storage capacity using this simple approach. The next degree of complexity is to reflect light off multiple apertures sequentially, which would linearly increase the path length by a factor proportional to the number of apertures. This approach remains insufficient to provide data storage capacities of interest. We have recently patented a breakthrough concept termed angle multiplexing (AM) that allows for a quadratic scaling of the achieved path length for a given number of apertures. ${ }^{13}$ Imagine two sets of apertures facing each other. Take 10 apertures on a side. If light travels in all the possible paths between those two sets of 10 apertures, we would have 200 paths traveled, because the light travels both directions. For a 100-m length between the two sets of apertures this would mean a $20-\mathrm{km}$ path length. Now, increase the number of apertures on each side to 100 . In that case, the distance would be $2000 \mathrm{~km}$, and with 1000 apertures on the side, the distance would be $200,000 \mathrm{~km}$. The increase in path length goes as the square of the number of apertures on a side.

$$
\text { Range }=2 L N^{2}
$$

where $L$ is the length between aperture arrays, and $N$ is the number of apertures on a side. This means very long path lengths can be used to store data. Certainly, the signal will need to be regenerated periodically, due to loss and dispersion of the signals. AM enables long paths in a 100-m long tube or even in a meter class vacuum cell. It also changes the approach to data storage in space. It is no longer best to move satellites as far apart as possible. Farther apart means larger and heavier apertures or aperture arrays. If the apertures are $1000 \mathrm{~km}$ apart, we can use many paths between them, and obtain a very long path. Storing for $1 \mathrm{~s}$ with a $100-\mathrm{m}$ long tube will require $3,000,000$ paths or 1225 apertures on a side, and with a 1000-km space between apertures will required 300 paths or $\sim 12$ apertures on a side. Longer storage times can be scaled from the above.

One more introduction subject-complexity. If we have $>16,000$ wavelength channels, and up to 50 spatial channels, two polarizations, and multiple gray scales per bit, we have a highly complex system. We will need to strive for simplicity as we develop the system. We are exploring simplification approaches.

\section{Enabling Technologies for LyteLoop Storage in Motion}

LyteLoop recognized early on that because the speed of light is high, it is not possible to store significant amounts of data using commonly deployed optical communications technologies. ${ }^{6}$ 
New paradigms were needed leading to new technologies to significantly extend the optical path length, reach data rates in $>100 \mathrm{~Pb} / \mathrm{s}$, and reduce power consumption.

\subsection{Angle Multiplexing}

Patented AM can significantly extend the path length between multiple free-space apertures, resulting in a longer distance a wave travels, and a longer period of storage for storage in motion applications. At a given data rate, this increases the amount of data that can be stored by the loop. A schematic of one implementation of AM is shown in Fig. 3. In this system, $M$ apertures in a plane (top side of Fig. 3) are facing $N$ apertures of another plane (bottom side of Fig. 3). Starting at the bottom left of the $N$ aperture plane, the laser beam travels to the aperture on the bottom left of the $M$ aperture plane and is reflected to another aperture in the $N$ aperture plane. It is then steered to another aperture of the $M$ aperture plane that sends it back to the initial aperture located at the bottom left of the $N$ aperture plane. At this location, light is sent to the next aperture in the upper $M$ aperture plane. By repeating this process, light travels to all the paths between all $M$ apertures of the top plane and two of the $N$ apertures of the lower plane. When the last aperture of the upper $M$ aperture plane is reached (located on the top-right), light is sent to a third aperture in the bottom $N$ aperture plane. The process is then repeated until the laser beam has traveled all possible paths between the $N$ apertures of the bottom plane and the $M$ apertures of the top plane. One can readily show that there are $M \times N$ paths in total. Hence, the path length was increased from $\min (M, N) \times L$ to $M \times N \times L$, where $L$ is the distance between the two planes. The above is an example path. We have explored and optimized the hop order for AM.

Our initial implementation of the AM concept used a combination of mirrors and volume holographic gratings (VHGs). A light ray is transmitted by a VHG unless it comes in at certain angle and wavelength, in which case it is reflected. Multiple angles can be embedded in a single VHG to steer the light in different directions according to the incoming angle of incidence. VHGs are diffractive elements consisting of a periodic phase or absorption perturbation, throughout the entire volume of the element. When a beam of incident light satisfies the Bragg phase-matching condition, it is diffracted by the periodic perturbation. Volume holograms were first treated by $\mathrm{H}$. Kogelnik in 1969 by the so-called coupled-wave theory. ${ }^{7}$ For volume phase holograms, it is possible to diffract $100 \%$ of the incoming reference light into the signal wave, i.e., full diffraction of light can be achieved. Moreover, by adequately designing the thickness of the VHG, low angular selectivity in the milliradian range can be obtained. However, VHGs possess a high dispersion: for a broadband signal with wavelengths spanning more than $100 \mathrm{~nm}$, it is necessary to employ dispersion-compensating components such as thin gratings. We have considered methods of making VHGs broadband enough for our application. Other approaches to design high angular selectivity apertures using low dispersive approaches for broadband operation are also currently pursued at LyteLoop. It will be easier to implement a broadband AM system if we can make it all reflective. Later we show a limited all reflective data storage system using AM.

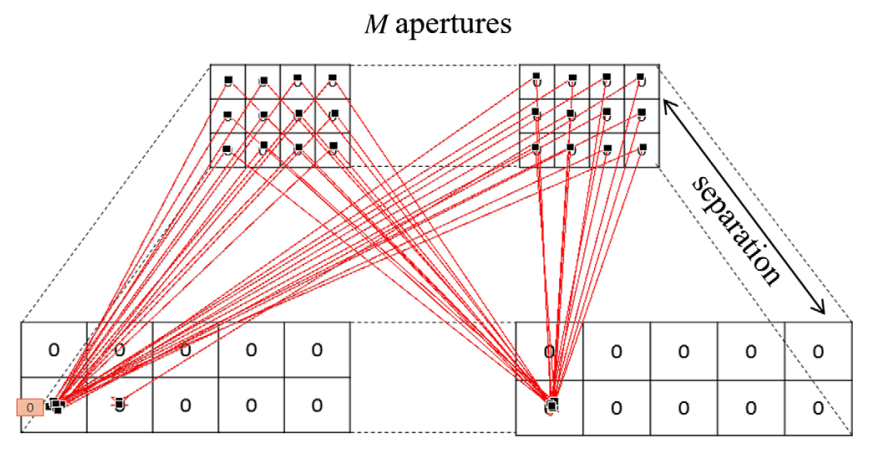

$N$ apertures

Fig. 3 Principle of AM between two planes of $M$ apertures on the top side and $N$ aperture on the bottom side. The red line represents the laser light travelling through the AM systems. For clarity, all the paths between 2 of the $N$ aperture side and all of the $M$ aperture side are represented. 


\subsection{Broadband Wavelength and Spatial Mode Division Multiplexing}

In LyteLoop fiber, using common fiber, we are limited to the near infrared (NIR) with wavelengths from 975 to $1800 \mathrm{~nm}$, we have shown that it is possible to reach a storage capacity from 2 to 10 petabytes using a fiber system of $10,000 \mathrm{~km} 50$ cores, 23 modes per core. ${ }^{8}$ In LyteLoop satellite, Lyteloop cell, and LyteLoop tube systems, storage capacity can be increased by extending the wavelengths of the lasers from NIR down to the visible and even UV light. Using wavelengths from 266 to $3400 \mathrm{~nm}$, we have a bandwidth of $1004 \mathrm{THz}$, which is many times more than in current fiber long haul systems. Using tens of layers of dielectric coating, broadband apertures with high reflectivity can be obtained, as shown in Fig. 4.

In addition to wider bandwidth, for free space, one can then use space-division-multiplexing using, e.g., OAM or HG modes, as discussed above. For fibers, we can also use spatial modes and multiple cores.

\subsection{Near Ideal All-Optical Regeneration}

In a storage in motion system based on laser communication, losses need to be compensated. Using state-of-the-art amplifiers poses two challenges: the first challenge is bandwidth, and the second is noise introduced by optical amplifiers exponentially increases the optical power consumption as the number of these amplifiers increases. In recent years, it was shown that using a chain of theoretical "ideal regenerators," it might be possible to significantly reduce the power consumption in long haul fiber systems. ${ }^{9}$ An ideal regenerator is a mathematically ideal device that has the following two properties:

1. Its nonlinear transfer function is a piece-wise function. The regenerator acts in a similar manner as a hard decision decoder. A noisy multilevel signal going through such a regenerator is transformed into a multilevel signal. In practice, nonlinear functions are approximations of a piece-wise function and such regenerators will be called near-ideal regenerators. ${ }^{10}$

2. All the pump power is transferred to the regenerated signal. We introduce the regenerator efficiency as the portion of the optical pump power that is effectively used to regenerate the signal in a piece-wise function type regenerator.

Optical coherent signals have both of their quadratures modulated. For this reason, a nearideal regenerator can either first separate each quadrature and separately regenerate their amplitude levels before recombining them, as shown in Fig. 5(a), or sequentially regenerate optical signal as in Fig. 5(b). Amplitude regenerators can be achieved using saturable amplifiers while

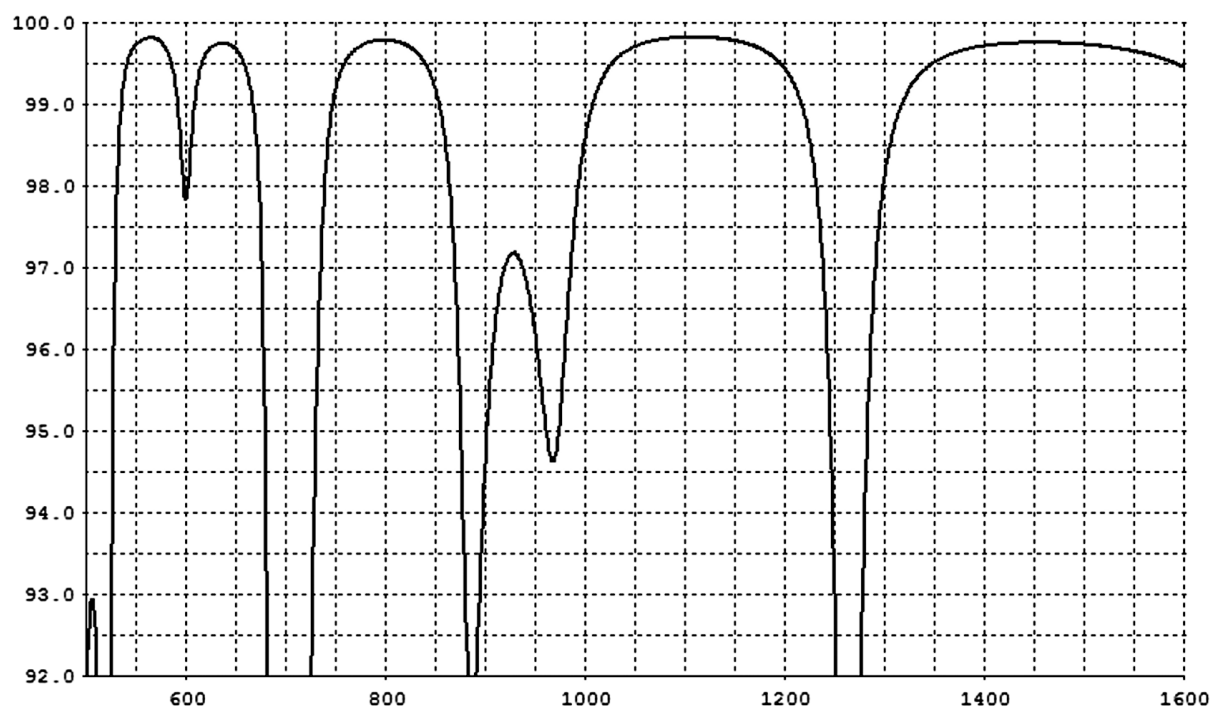

Fig. 4 Mirror reflectivity as a function of wavelength. 


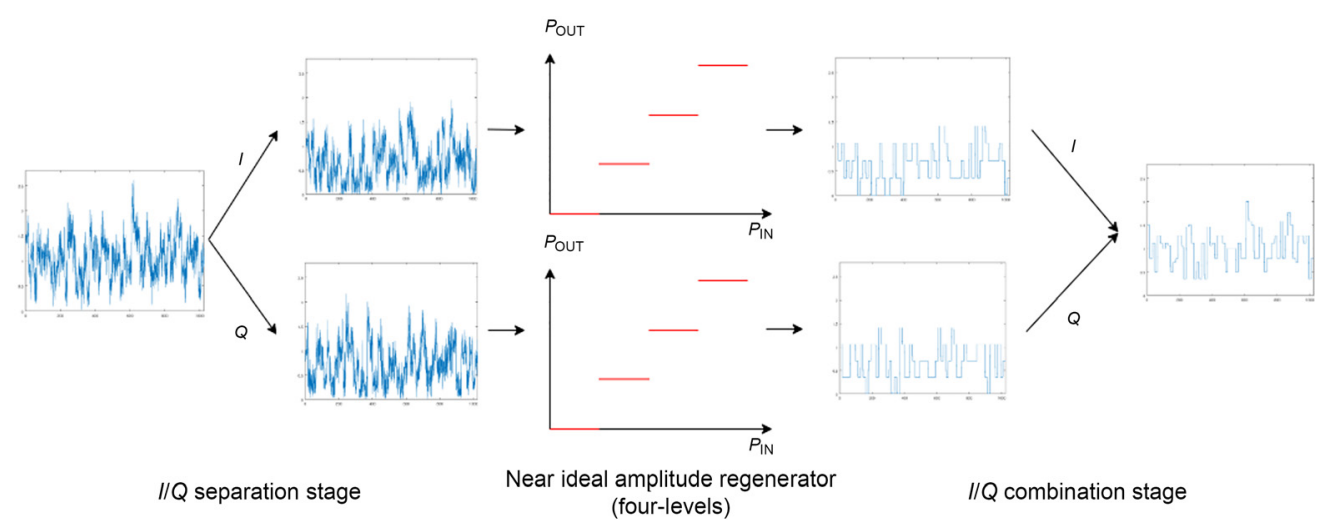

(a)

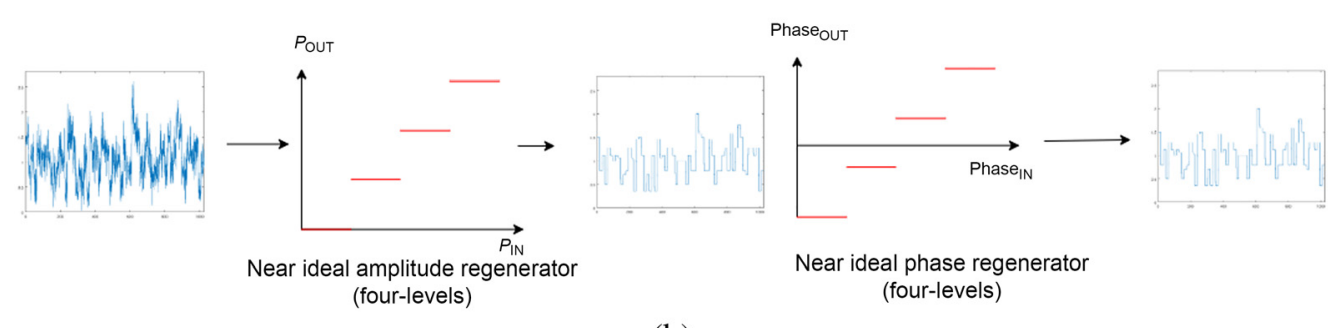

(b)

Fig. 5 Principle of a near-ideal regeneration for a coherent optical signal where (a) quadratures are separately regenerated and recombined and (b) amplitude is first regenerated then phase is regenerated.

phase-sensitive amplification can be used to regenerate phase. In LyteLoop fiber, LyteLoop cube, and LyteLoop tube systems, the controlled environment and the fact that all regenerators are in the same place can be harnessed to increase the efficiency and near ideality of the regenerators. Using our patent-pending concepts of sharing components and functionalities that are located in one place, we are developing regenerators with the potential of being scaled to larger numbers of spatial and wavelength channels [space division multiplexing (SDM)-wavelength-division multiplexing (WDM)], with the ability to share the pump and hence its coherence among signals and near-ideal regenerators.

To illustrate the gain in optical power consumption, we have calculated the theoretical lower limit of optical power consumption for a LyteLoop fiber system at the quantum Shannon limit. The fiber under consideration has 50 cores and 23 spatial modes per core and a total length of $10,000 \mathrm{~km}$. We assumed its absorption is the same for all modes and all cores. It is shown in Fig. 6(a) with a minimum loss of $0.18 \mathrm{~dB} / \mathrm{km}$ at $1550 \mathrm{~nm}$. The results are shown in Fig. 6(b) for ideal regenerators positioned at different spans. For each number of bits/symbol, the bandwidth of the optical signal was adjusted to reach a total capacity of 0.98 petabytes. The results show that it is possible to reduce the theoretical minimum optical power of keeping data in motion to below 10 mwatts/Tbyte with the right spacing and coding design.

We have calculated that similar optical power reduction can be obtained in LyteLoop cell, LyteLoop tube, and LyteLoop satellite. It is important to note that these savings occur if the optical signal is not detected in the electric domain. In other words, as long as read/write operations in the storage in motion system do not occur, it is possible to have a low idle power consumption.

\section{Experiments Accomplished}

\subsection{Fiber-Based Proof of Concept}

Using state-of-the-art long-haul telecommunications systems, we have set up a storage loop with 1.5 -Gbytes capacity. The loop is composed of a point-to-point 1000-km long-haul WDM 


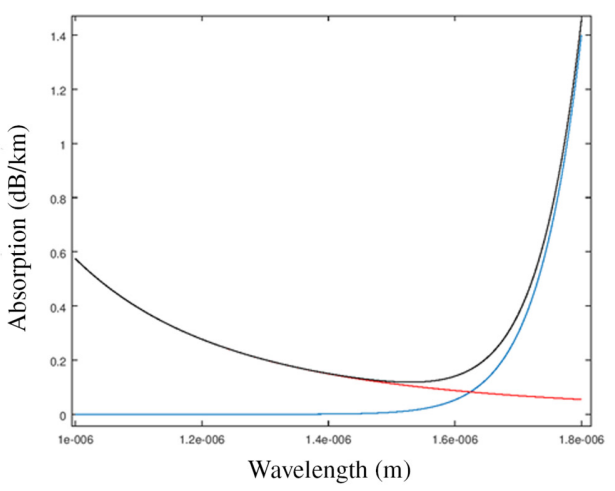

(a)

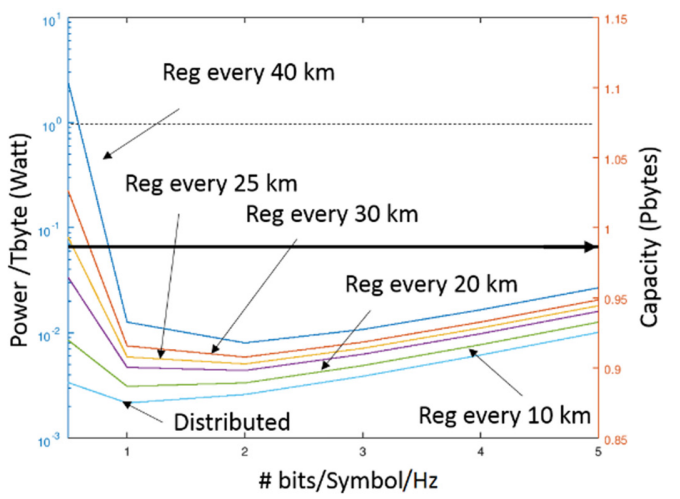

(b)

Fig. 6 (a) Calculated absorption spectrum of a silica based multicore and multimode fiber with $0.18 \mathrm{~dB} / \mathrm{km}$ at $1550 \mathrm{~nm}$ where all modes are supposed to have the same theoretical absorption. The black curve is the total absorption, the red curve the Rayleigh contribution and the blue curve the NIR absorption. (b) Power consumption (left vertical axis) and capacity (right vertical axis) as a function of \#bits/Symbol/Hz for different spacing between ideal regenerators. The capacity is kept constant at 0.98 Petabytes while the bandwidth is reduced.

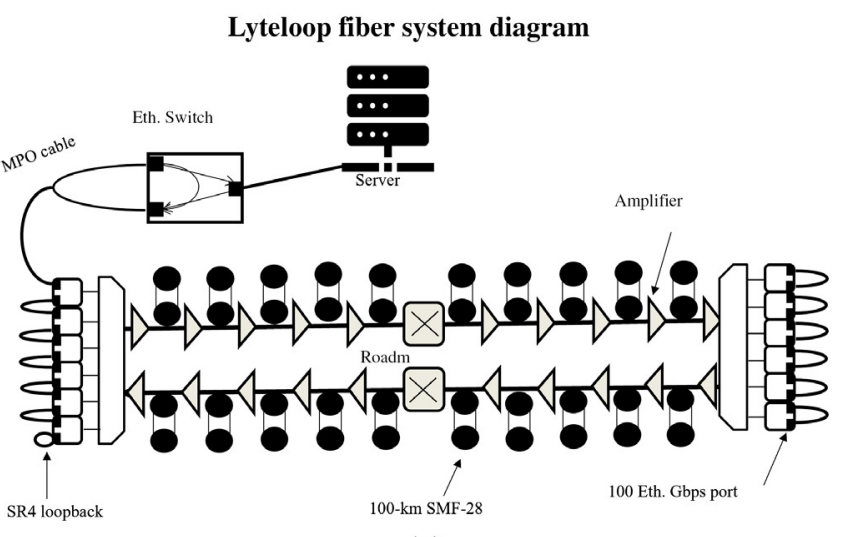

(a)

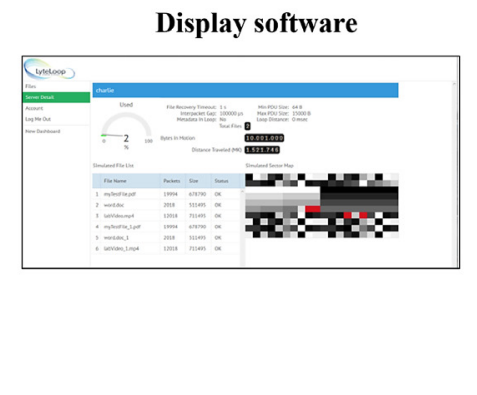

(b)

Fig. 7 (a) Storage in a 2000-km fiber loop using a long-haul bidirectional 1000-km WDM system. Eth: ethernet (b) graphic interface for storage system access and monitoring.

coherent transmission system as shown in Fig. 7(a). Each terminal consists of six transponders that convert $2 \times 100$ gpbs ethernet stream onto one 200 gbps optical transport unit, stream carried by a dual-polarization 16-QAM modulated C-band WDM 37.5-GHz channel. All six WDM channels are multiplexed into the transmission line that is composed in each direction of 10 spans of 100-km SMF-28 with amplifiers and ROADMs in between the spans for signal amplification and WDM channel equalization. Excluding 25\% forward error correction overhead, an aggregated of $1.2 \mathrm{Tbps}$ is propagated over $2 \times 1000 \mathrm{~km}$ in both direction, which corresponds to a storage capacity of 1.5 Gbytes. To write a file, a proprietary algorithm converts the file to be stored in the loop to $100 \mathrm{Gbps}$ ethernet packets that are fed through an ethernet switch to one of the terminal ethernet port. By connecting all the other ethernet ports in a daisy-chain configuration using ethernet cables, the $100 \mathrm{Gbps}$ stream is propagated 12 times back and forth over the $1000-\mathrm{km}$ transmission system. At the last port, a loopback is used to propagate the stream back to the input ports. In this manner, the whole capacity of the storage loop is filled with only a 100-Gbps line feed, without the need to generate a 1.2 Tbps data stream. When the stream has looped back to the first ethernet port, it is sent to the second port of the ethernet switch using a custom-made multi-fiber push-on cable (ribbon cable supporting multiple fibers) to separate the ethernet ingress and egress streams from/to the transponder to/from the switch. 
By default, the switch will redirect the stream to the port that is connected to the transponder input port.

When the user decides to access a file, the switch reroutes a copy of the packets corresponding to the desired file to the server. It is also possible to erase any file by disabling the switch rerouting to the storage loop. A user interface and monitor dashboard were created to seamlessly perform any disk operation on the storage loop and monitor the storage space already used and the storage space still available [Fig. 7(b)]. It is noteworthy that in this system, parity-check is performed at every loop. Files were stored for over months with no errors, corresponding to a distance traveled of nearly 300 billion $\mathrm{km}$.

\subsection{Free-Space Prototype}

A free-space prototype is currently being built. At completion, it will consist of a transmitter system having 70 wavelength channels spanning from 550 to $1550 \mathrm{~nm}$. Each wavelength will be composed of 10 spatial channels, either OAM modes or HG modes. In total, 700 channels at 200 Gbps will be generated for an aggregate throughput of 140 terabits per second.

The optical path will be based on the patented AM system with 40 apertures facing 60 apertures 10-m apart. The total optical path will therefore be $48 \mathrm{~km}$ since both directions of propagation will be used.

The free-space prototype will be built in four phases. In phase 1, one-dimensional (1D) AM has been demonstrated using two different approaches. The later phases concentrate on generating more wavelengths and storing them in AM setups with increased optical path length. This paper focuses on our experimental results in phase 1.

The group has demonstrated the feasibility of the transmitter system design, by connecting $10 \mathrm{C}$-band channel transceivers propagating through $10 \mathrm{HG}$ modes and back. The SNR was calculated per addition of mode and per channel. Figure 8 shows a schematic of the experimental set up.

Figure 9 shows that the SNR reduces with the addition of HG modes. This can be attributed to crosstalk between the spatial modes. The SDM devices are sensitive to alignment and vibrations. With better alignment and vibration isolation techniques, we expect these SNR values to improve.

Figure 10 shows the variation in SNR with channel frequency. In this experiment, all 10 spatial modes were sourced from 12 unique C-band channels and were split into 10 copies using

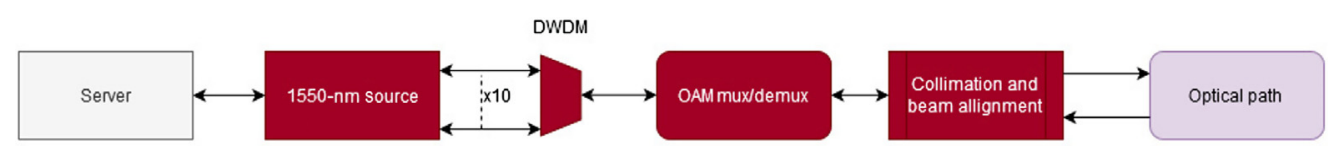

Fig. 8 Schematic showing experiment conducted using 10 DWDM channels and 10 OAM modes.

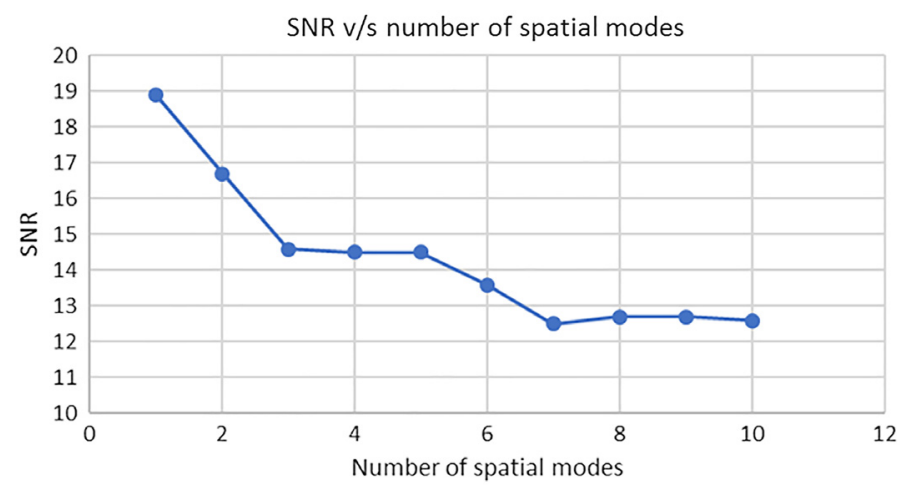

Fig. 9 Graph showing how the SNR changes with the addition of HG modes. 


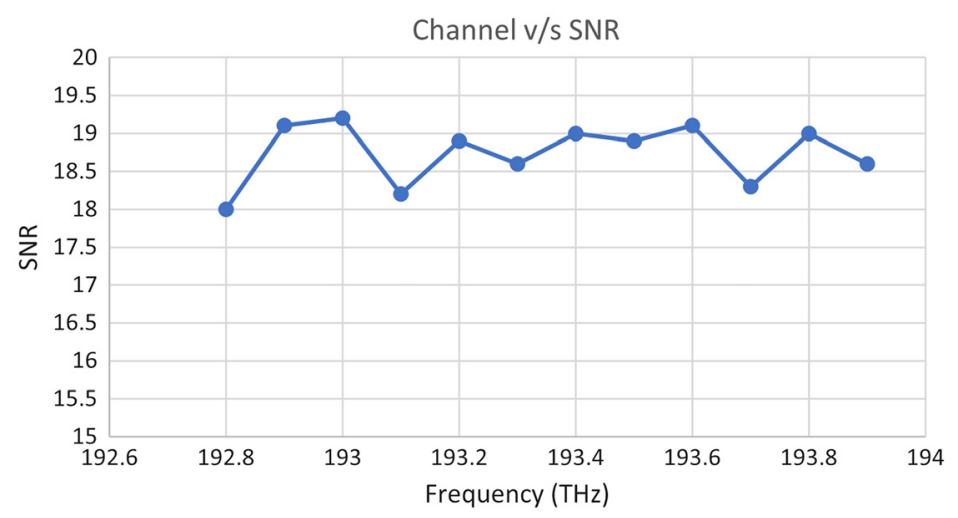

Fig. 10 Graph showing the variation of SNR with channel frequency.

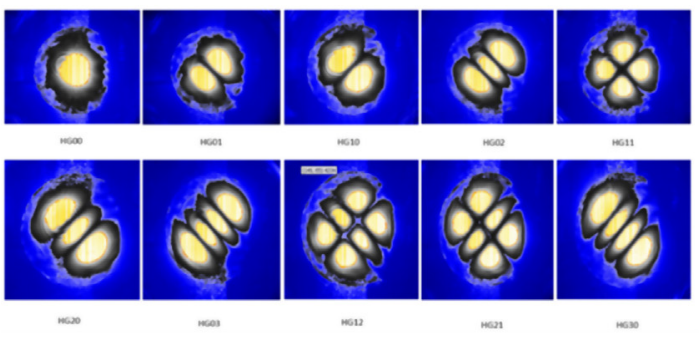

(a)

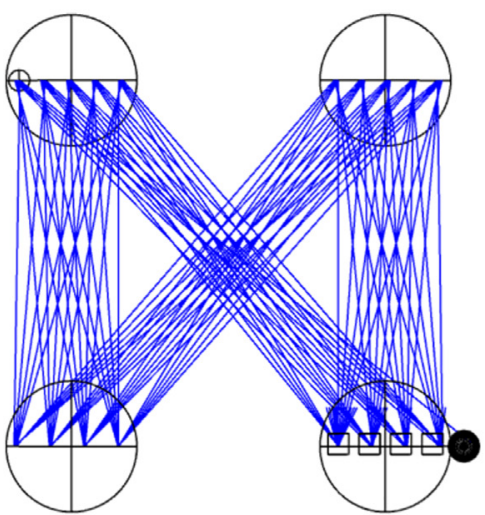

(b)

Fig. 11 (a) IR CCD camera images of the HG modes generated at $1550 \mathrm{~nm}$ by the free-space LyteLoop prototype. (b) Ray-tracing simulation of the AM system with eight apertures on each side (64 optical paths).

a $1 \times 10$ optical splitter. As can be seen, the SNR is above 18 and consistent through the 10 channels. This shows that the SDM device is capable of producing HG modes consistently over 10 C-band channels

Figure 11(a) shows the HG modes generated by the prototype system at $1550 \mathrm{~nm}$, recorder using an IR CCD camera. Figure 11(b) shows a ray-tracing simulation of part of the AM system.

One of the critical aspects of phase 1 was demonstrating AM in $1 \mathrm{D}$, meaning all of the apertures are in a single plane. In phase 2, we have apertures in two planes, so arrays on each side will become two dimensional (2D). In phase 1, we have two lines of apertures on each side. The achieved goals of phase 1 are shown in Table 2. Initially, we demonstrated AM using a combination of VHGs and mirrors. One issue with this approach is the VHGs are not broadband, but we will have to use wavelength channels at multiple wavelengths. Then, because of the interest in broadband operation, we also demonstrated 1D AM using mirrors.

There were three-AM demonstrations completed in the lab for phase 1. The first (phase 1a) used VHGs and had 1D aperture arrays on each side, the second (phase 1b) used VHGs and had two-dimensional aperture arrays on each side, and the third used only mirrors and had 1D aperture arrays on each side. All three demonstrations to be discussed were done with a 633-nm Gaussian beam with the intention of adding more wavelengths and spatial modes in future phases. The objective of these lab demonstrations was to reduce risk for an eventual LyteLoop product. The geometry of the AM subsystem is such that this reduces more risk for tube and cell than it does for space, because the experiment we conducted was more similar to the tube or cell, geometry. 
Table 2 Overview of results achieved in the three-AM demonstrations.

\begin{tabular}{lccc}
\hline \hline & Phase 1a & Phase 1b & $\begin{array}{c}\text { All mirror angle } \\
\text { multiplexing (AMAM) }\end{array}$ \\
\hline Unidirectional/bidirectional & Unidirectional & Unidirectional & Bidirectional \\
Active apertures (apertures using VHGs) & 4 & 2 & 0 \\
Side A array size & $8 \times 1$ & $\begin{array}{c}\text { Four apertures in different } \\
\text { rows and columns }\end{array}$ & $8 \times 1$ \\
Side B array size & $10 \times 1$ & $\begin{array}{c}\text { Four apertures in different } \\
\text { rows and columns }\end{array}$ & $6 \times 1$ \\
Total apertures & 18 & 8 & 14 \\
Number of paths & 80 & 16 & 98 \\
Cavity length $(\mathrm{m})$ & 10 & 10 & 10 \\
Total optical path length $(\mathrm{m})$ & 800 & 160 & 980 \\
\hline \hline
\end{tabular}

\subsubsection{Phase 1a and phase 1b-angle multiplexing with VHGs}

VHGs were chosen for phase $1 \mathrm{a}$ and $1 \mathrm{~b}$ because of their physical properties of angular selectivity and diffraction efficiency. The optical path length of a laser in a $10-\mathrm{m}$ long modified $4 \mathrm{f}$ cavity was successfully extended to $800 \mathrm{~m}$ with the phase $1 \mathrm{a}$ approach and $160 \mathrm{~m}$ in the phase $1 \mathrm{~b}$ approach. The cavity utilized two large, curved mirrors (mirrors 1 and 3) on side A and another two large, curved mirrors (mirrors 2 and 4 ) on side B. Many of the apertures were simply virtual spots on these large mirrors that the light repeatedly bounced off. VHGs and a flyback mirror were mounted in front of the large mirrors to create the necessary propriety geometry for AM to function properly. For phase 1b, two 1D VHGs were mounted diagonally, and orthogonal to each other, at both mirror 1 apertures to effectively steer the light in 2D. All VHGs for both phase 1a and $1 \mathrm{~b}$ were aligned for maximum throughput and the correct angular separation using piezoelectric motors. Figure 12 shows AM demonstrated experimentally, using mirrors and VHGs, in phase 1a.

A critical aspect of any AM setup is signal loss, since after roughly $20 \mathrm{~dB}$ of loss signal is unable to be read by our current transceivers. Maintaining high mirror reflectivity is thus crucial. Transmissive VHGs were shown to have $\sim 5 \%$ loss per pass, which severely lowered transmission after many bounces through the system. This loss was measured for phase 1a and is shown in Table 3.

Figure 13 shows a demonstration of AM using gratings.

\subsubsection{Phase 1-All mirror angle multiplexing}

A new geometrical approach to AM that only uses mirrors, no gratings, was designed and demonstrated in the lab after phase 1a and phase 1b were complete. Unlike the VHG demonstrations, it was inherently broadband and bidirectional, and it had eight apertures on one side and six on the other for a total of 98 hops. It also had less loss. The same four large, curved mirrors from phase $1 \mathrm{a}$ and phase $1 \mathrm{~b}$ were used in a similar axial and radial orientation with several smaller mirrors added to redirect the light at certain locations.

The input power was $16.20 \mathrm{mw}$ and the power at the egress was $3.08 \mathrm{mw}$ for a transmission of $19 \%$. All the loss came from the scattering and absorption of the mirrors. The system had a total of 98 hops. This can be calculated by Hops $=2 *\left(M_{T o t} N_{T o t}\right)+2$ where $M_{T o t}$ and $N_{T o t}$ are the total number of apertures on each side. The additional two hops come from the two ingress hops that were not counted before the input into the AMAM system. Mirrors 1 and 3 had four apertures each and mirrors 2 and 4 had three apertures each, so there are double that many apertures on each side, and thus there are $2 *(8 * 6)+2=98$ Hops. Figures 14 and 15 


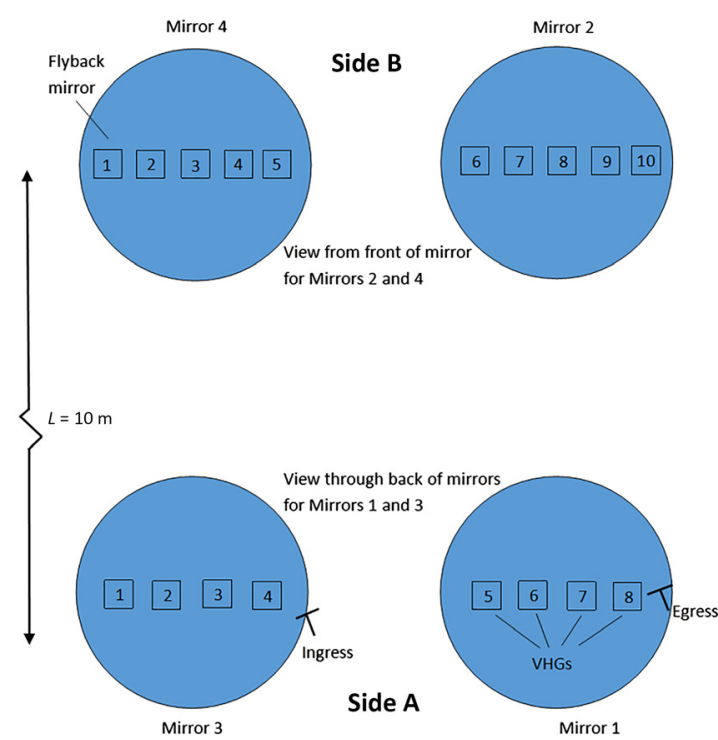

(a)

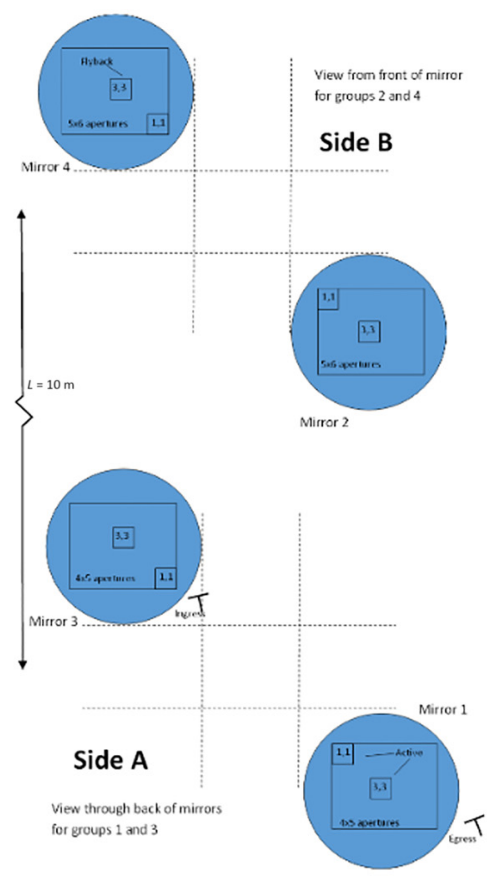

(b)

Fig. 12 (a) Experimental setup for phase 1a with VHGs. (b) Experimental setup for phase 1b with VHGs.

Table 3 Transmission power loss during propagation due to mirror and grating losses for phase $1 \mathrm{a}$.

\begin{tabular}{lllc}
\hline \hline Location & $\begin{array}{c}\text { Power } \\
(\mathrm{mw})\end{array}$ & $\begin{array}{c}\text { Power } \\
(\mathrm{dBm})\end{array}$ & $\begin{array}{c}\text { Net transmission } \\
(\%)\end{array}$ \\
\hline After the ingress beam expander & 15.34 & 11.86 & 100 \\
At mirror 3, aperture 1 after first grating alignment & 13.47 & 11.29 & 87.8 \\
At mirror 3, aperture 2 after second grating alignment & 6.60 & 8.20 & 43 \\
At mirror 3, aperture 3 after third grating alignment & 3.19 & 5.04 & 20.8 \\
At mirror 3, aperture 4 after fourth grating alignment & 1.59 & 2.01 & 10.4 \\
At mirror, after egress mirror and steering mirrors & 0.80 & -0.97 & 5.2 \\
\hline \hline
\end{tabular}

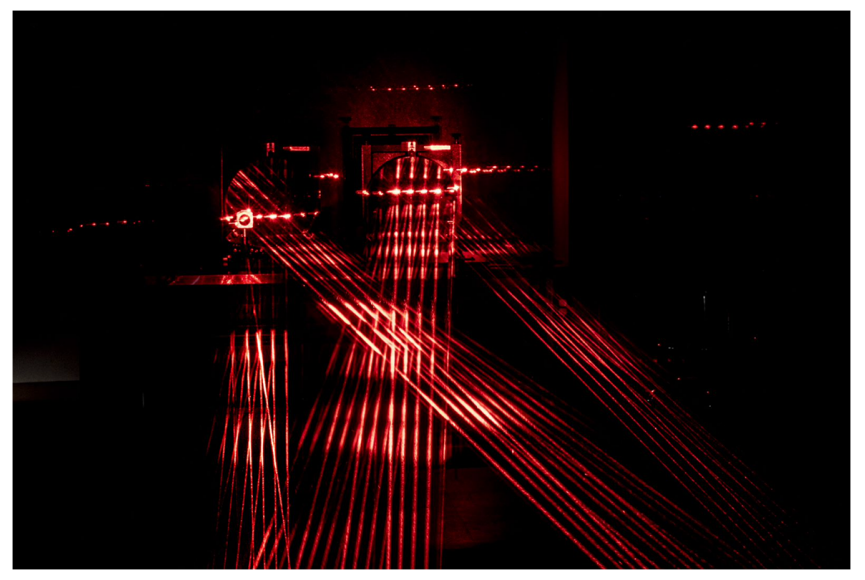

Fig. 13 Phase 1a in operation. 


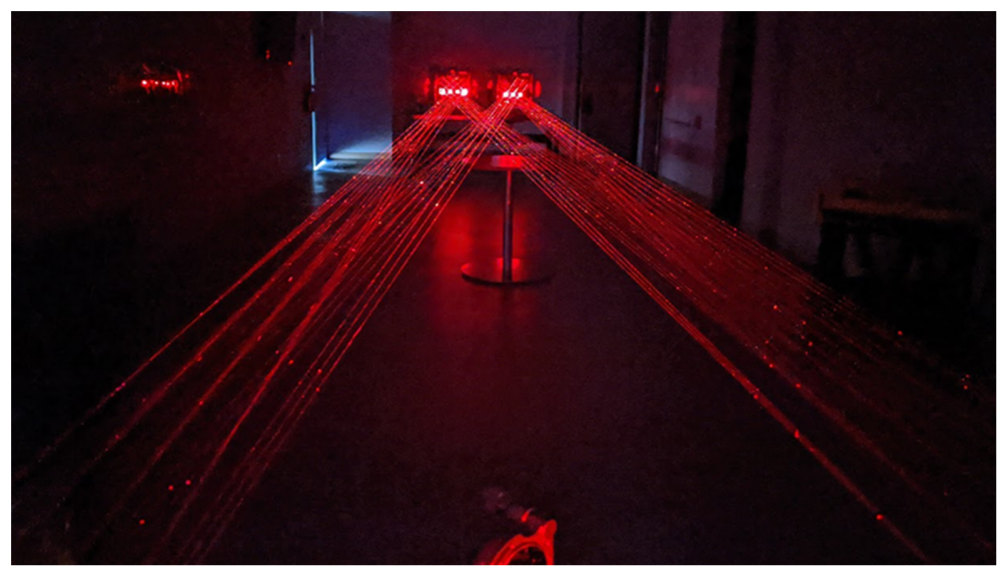

Fig. 14 AMAM as demonstrated in the lab as seen from the mirror 1, mirror 3 side. Mirror 2 is on the right and mirror 4 is on the left.

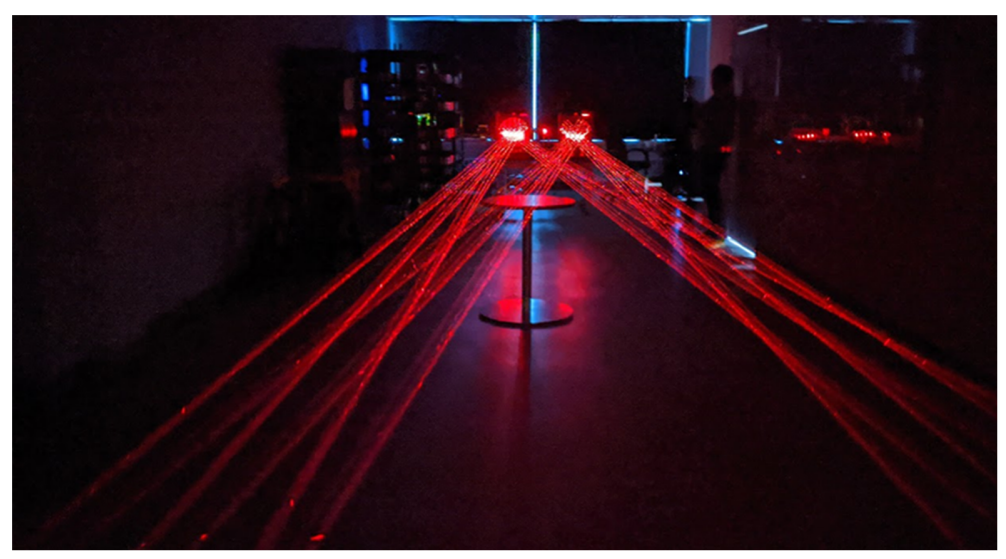

Fig. 15 AMAM as demonstrated in the lab as seen from the mirror 2, mirror 4 side. Mirror 1 is on the left and mirror 3 is on the right.

show AM operating using mirrors. It is noteworthy that the number of hops can readily be increased by modifying the inclination angles of the ingress/egress mirrors as well as that of the mirrors.

\section{Conclusions}

We have reviewed the concepts of storage in motion that are driving the development of LyteLoop. We then discuss the required components, and the experiments we have conducted to date. After successfully building the fiber proof of concept using off-the-shelf long-haul fiber telecommunications equipment, a free-space prototype was built in 1D with a limited number of wavelengths. AM has been demonstrated using two different methods, one of them easily scalable to broadband operation. The planned free-space experiments will have an aggregated throughput of 140 Tbps spanning the visible and NIR wavelengths.

By scaling the technologies currently developed at LyteLoop such as AM, near-ideal regeneration, and bandwidth extension to the visible and UV wavelengths, we believe it will be possible to develop data centers capable of storing more than an exabyte of data in motion with $100 \mathrm{~s}$ of $\mathrm{Pb} / \mathrm{s}$ of throughput. These data centers will be able to accommodate the exponential growth of data generation throughout the world and have other advantages. This is the first reporting for many of these experiments. 


\section{References}

1. D. M. Damaghi et al., "Data in motion storage system and method," US Patent US20170280211.

2. D. M. Damaghi et al., "Data in motion storage system and method," US Patent US20190166414.

3. C. Krittanawong, "The rise of artificial intelligence and the uncertain future for physicians," Eur. J. Int. Med. 48, e13-e14 (2018).

4. M. Padgett et al., "Divergence of an orbital-angular-momentum-carrying beam upon propagation," New J. Phys. 17, 023011 (2015).

5. M. Koshiba, K. Saitoh, and Y. Kokubun, "Heterogeneous multi-corefibers: proposal and design principle," IEICE Electron. Express 6(2), 98-103.

6. P. F. McManamon et al., "Lyteloop data "storage in motion" using high-bandwidth laser communications," Proc. SPIE 10947, 109470E (2019).

7. H. Kogelnik, "Coupled-wave theory for thick hologram gratings," Bell Syst. Tech. J. 48, 2909 (1969).

8. T. Morioka et al., "R\&D of 3M Technologies towards the realization of exabit/s optical communications," IEICE Trans. Commun. E100.B(9), 1707-1715 (2017).

9. C. Antonelli et al., "Quantum limits on the energy consumption of optical transmission systems," J. Lightwave Technol. 32(10), 1853-1860 (2014).

10. M. Sorokina and S. Turitsyn, "Regeneration limit of classical Shannon capacity," Nat. Commun. 5, 3861 (2014).

11. J. Wang et al., "Terabit free-space data transmission employing orbital angular momentum multiplexing," Nat. Photon. 6(7), 488-496 (2012).

12. N. Bozinovic et al., "Terabit-scale orbital angular momentum mode division multiplexing in fibers," Science 340(6140), 1545-1548 (2013).

13. P. F. McManamon et al., "System and method for extending path length of a wave signal using angle multiplexing," US20200050389A (2020).

Paul McManamon was chief scientist of the Air Force Research Laboratory (AFRL) Sensors Directorate until 2008. He is president of Exciting Technology, technical director of Lidar and Optical Communications Center, and chief scientist for LyteLoop. He chaired the National Academy of Sciences, Laser Radar (2014), was co-chair of Optics and Photonics (2012), and vice chair of the 2010 Seeing Photons. He is a fellow of SPIE, IEEE, OSA, AFRL, DEPs, MSS, and AIAA, and was president of SPIE in 2006.

Armand Vedadi received his $\mathrm{PhD}$ in electrical engineering from the Femto-ST Institute of the University of Franche-Comte. He has held research positions in various academic institutes around the world. Since 2015, he has been involved with startups in photonics and is currently a senior R\&D engineer at LyteLoop. His research interests involve optical communication, information theory, nonlinear optics, and photonic processing devices. He has published dozens of journal and conference papers and holds a few patents.

Alan E. Willner holds the Steven and Kathryn Sample Chair in Engineering at the University of Southern California. Honors include: Member National Academy of Engineering; International Fellow UK Royal Academy of Engineering; IEEE Summer Award; NSF Presidential Faculty Fellows Award from White House; Fulbright, Guggenheim, Packard Fellowships; OSA Forman Engineering Excellence Award; and SPIE President's Award. He was cochair National Academies' Study on Optics \& Photonics; President Optical Society and IEEE Photonics Society; and Editor-in-Chief of Optics Letters and J. Lightwave Technology. Fellow IEEE, OSA, SPIE.

Dipayan Choudhary is an optical engineer working at LyteLoop. He graduated from New Jersey Institute of Technology with a master's in science in renewable energy, with a concentration on semiconductor physics. He has experience in lasers, light emitting diodes, and optical communication networks. He has been a part of LyteLoop since 2017 and is a part of LyteLoop's research and design team. 
Nick Montifiore is an optical engineer who studied optical engineering at the University of Rochester. He has experience in optics and physics research, lens design, and production engineering. He has been at LyteLoop since 2019 and has focused on design and lab work on LyteLoop's patented AM techniques.

Ohad Harlev is CEO of LyteLoop. He was president of RRsat America, and earlier served as RRsat's managing director, corporate general counsel, and company secretary. He held senior positions as VP, business development and general counsel of Kamor Ltd. (TASE: KMOR); and at the fiber optics division of Gilat Satcom (TASE and AIM); and corporate counsel at Amdocs (NASDAQ: DOX). He has extensive experience as a leader in hi-tech fields including telecommunication, fintech and satellites. 